\title{
Organ Model
}

National Cancer Institute

\section{Source}

National Cancer Institute. Organ Model. NCI Thesaurus. Code C19689.

Use of in vitro tissues or organ explants as surrogates for whole organism testing. 